\title{
Damage Creep Analysis and Instability Delay Time of Ultrahigh pillar considering Initial Transverse Deformation
}

\author{
Qi Wei ${ }^{1,2}$, FU Jian-xin ${ }^{1,2,{ }^{*}}$, and SONG Wei-dong ${ }^{1,2}$ \\ ${ }^{1}$ School of Civil and Resource Engineering, Beijing 100083, China \\ ${ }^{2}$ State Key Laboratory of High-Efficient Mining and Safety of Metal Mines, Ministry of Education, Beijing 100083, China
}

\begin{abstract}
According to the ultrahigh pillar structure and mechanical characteristics, considering the initial geometric deviation caused by mining disturbance, the simplified model of the pressure bar is established. Based on elastic-plastic theory, the deflection and internal forces of ultrahigh pillar with initial geometric imperfections are analyzed and discussed. Based on the Kachanov theory of damage, the damage of ultrahigh pillar is analyzed, the damage failure time is calculated. The results show that under the influence of the initial deflection, pillar will appear different degree of additional deflection, which grows with the increase of initial deflection and pressure ratio. Analysis of engineering examples show that the start time of damage and instability of delay time of pillar has close relationship with initial deflection, pressure ratio and compressive strength. With the increase of pressure ratio and initial deflection, the damage incubation time and the instability of delay time quickly become small, the greater the compressive strength, the greater the instability of the delay time, which means that the pillar is more stable. The results of this study provide a theoretical basis for stability analysis and control of ultrahigh pillar..
\end{abstract}

\section{Introduce}

Stability analysis of mined out area has always been a hot issue of [1] - [4], and also an extremely complex problem. The structure of mined-out area is an important influence factor of stability [5]. The pillar instability is one of the main forms of mined-out area damage.

At present, a great deal of domestic and foreign literatures has already obtained abundant fruits on the research of pillar stability of mined-out area [6] - [8] and many scholars put forward a variety of theoretical models, such as the rheological model of roof-pillar system [9], nonlinear dynamics model of shell [10]. Li Hong [11] derived the stable model of pillar in asymmetric mining, the theoretical solution is obtained. Exadaktylos G [12] studied the pillar instability phenomenon caused by the axial splitting under the uniaxial pressure based on the fracture mechanics. Yin Shenghua [13] evaluated the sensitive of influence factors on the stability of pillar using the method of range analysis in orthogonal. The current study comprehensively reveals the stability characteristics and instability mode of pillar with large width-height ratio. However, the study of the stability of ultrahigh pillar with small width-height ratio relatively few.

With the increase of mining depth, due to the deterioration of the mining environment, safe and efficient subsequent filling method will become the main mining method [14]. When the surrounding rock conditions are good, the stage room with large height and subsequent filling will become the preferred mining method while a lot of ultrahigh pillars will appear at the same time. The pillar is the key factor to control the stope stability. Because height is far greater than the width, so the overall stability of ultrahigh pillar in the transverse direction is significantly decreased. The deformation characteristics of ultrahigh pillar are similar to that of pressure bar structure. But due to the complexity and the anisotropy of the occurrence environment and rock properties, ultrahigh pillar instability mechanism is more complex. In addition, there has been a certain degree of transverse deformation in the ultrahigh pillar caused by the blasting in the mining process, which has important effect on the stability of ultrahigh pillar. However, the study of the stability of pillar with initial transverse deformation is still less.

In this paper, based on the plastic-elastic theory, we analyzed the stability of ultrahigh pillar with transverse initial deformation firstly and established the computing model. Then, based on the creep damage theory, we analyzed damage process of ultrahigh pillar and obtained the instability time of ultrahigh pillar.

\section{Simplified mechanical}

Figures and tables, as originals of good quality and well contrasted, are to be in their final form, ready for reproduction, pasted in the appropriate place in the text. Try to ensure that the size of the text in your figures is approximately the same size as the main text (10 point). Try to ensure that lines are no thinner than 0.25 point.

\footnotetext{
* Corresponding author: fujun0011@,126.com
} 


\subsection{Elastic-plastic strut model of ultrahigh pillar}

Ultrahigh pillar of underground mine is often under high pressure which resulted in a large longitudinal deformation in the axial direction which is fa greater than horizontal deformation that can be ignored. So, the top of the pillar can be simplified as a directional support with only axial deformation. At the bottom of the pillar, because the stiffness of floor is far greater than that of pillar, the angular displacement and linear displacement can be ignored. So the bottom of pillar can be simplified as fixed bearing. The Elastic-plastic strut model of ultrahigh pillar is shown in Fig. 1 .

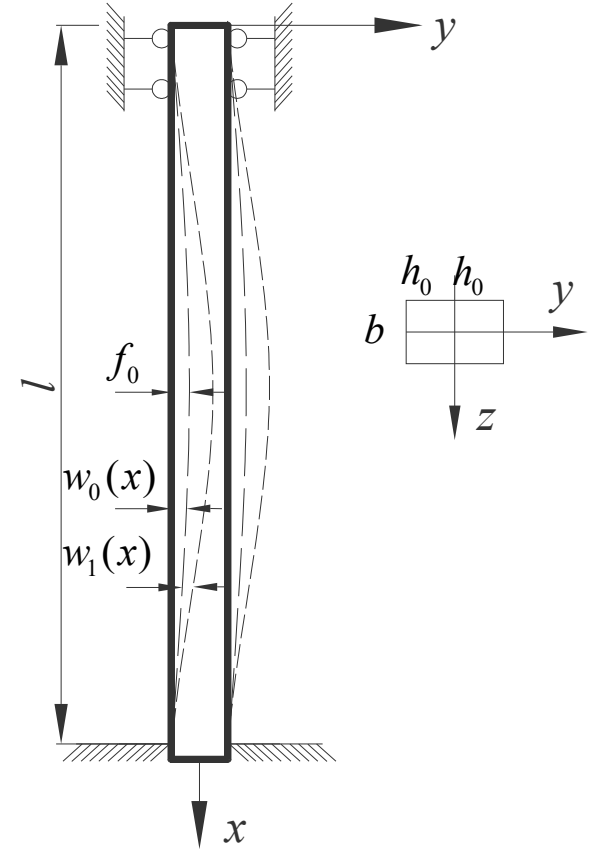

Fig. 1. Simplified strut model of ultrahigh pillar

As shown in Fig.1, the height of pillar is $l$, the length of $\mathrm{y}$-direction is $2 h_{0}$, the length of z-direction is $b$. After the formation of the pillar, the initial transverse deformation of pillar is $w_{0}(x)$, the initial transverse deformation in the middle is $f_{0}$. The transverse deformation caused by the overlying load on the top of pillar is $w_{1}(x)$.

\subsection{Basic assumptions}

In order to simplify the analysis process, the following basic assumptions is done in the calculation:

(1) The weight of the pillar is not considered;

(2) Before entering the stage of crack extension, the deformation of pillar is small and overlying load did not change significantly;

(3) In the mining process, rock plastic deformation did not change the constraint type of both ends of pillar;

(4) In the process of damage, the elastic modulus and inertia moment of cross section of pillar maintained which meet the plane section assumption.
In order to simplify the analysis, the influence of blasting vibration and horizontal stress are neglected, the effect of vertical load is only considered.

\subsection{Analysis of deformation and stress of pillar}

(1) Transverse deformation analysis

For the mechanical model shown in Figure 1, the boundary conditions are expressed as:

$$
\left\{\begin{array}{l}
\left.w(0)\right|_{x=0}=0,\left.w(l)\right|_{x=l}=0 \\
\left.w(0)\right|_{x=0}=0,\left.w(l)\right|_{x=l}=0
\end{array}\right.
$$

Assume that the initial transverse deformation is

$$
w_{0}(x)=f_{0}\left(1-\cos \frac{2 \pi x}{l}\right)
$$

The transverse deformation caused by the overlying load on the top of pillar is $w_{1}(x)$, then the transverse deformation of pillar can be obtained as

$$
w(x)=w_{0}(x)+w_{1}(x)
$$

According to the theory of strut, Eq. (3) satisfies the differential equation as shown Eq. (4) :

$$
E I \frac{\partial^{4} w_{1}(x)}{\partial x^{4}}+P \frac{\partial^{2} w(x)}{\partial x^{2}}=0
$$

Where, $E$ is elastic modulus, $I$ is inertia moment, $\mathrm{P}$ is overlying load. Taking Eq. (2) and Eq. (3) into Eq. (4), then we can be obtained as

$$
E I \frac{\partial^{4} w_{1}(x)}{\partial x^{4}}+P \frac{\partial^{2} w(x)}{\partial x^{2}}+\frac{4 \pi^{2} P f_{0}}{l^{2}} \cos \frac{2 \pi x}{l}=0
$$

The transverse deformation $w_{1}(x)$ can be obtained as:

$$
\begin{aligned}
w_{1}(x)= & a \sin \sqrt{\frac{P}{E I}} x+b \cos \sqrt{\frac{P}{E I}} x+c \frac{x}{l}+d+ \\
& \frac{P f_{0}}{2 P-\frac{8 \pi^{2}}{l^{2}}} \cos \frac{2 \pi x}{l}
\end{aligned}
$$

Where, $a, b, c, d$ are constants.

Taking Eq. (1) into Eq. (6), we can obtain as

$$
w_{1}(x)=\frac{P}{P_{c r}-P} f_{0} \cdot\left(1-\cos \frac{2 \pi x}{l}\right)
$$

Where, $P_{c r}$ is the limit load of pillar. For the strut model in this paper, $P_{c r}=\frac{4 \pi^{2} E I}{l^{2}}$.

The total transverse deformation can be calculated as

$$
w(x)=w_{0}(x)+w_{1}(x)=\frac{P_{c r}}{P_{c r}-P} f_{0} \bullet\left(1-\cos \frac{2 \pi x}{l}\right)(8)
$$

Assuming $\alpha=P / P_{c r}$, then Eq. (8) can be converted to

$$
w(x)=\frac{f_{0}}{1-\alpha} \cdot\left(1-\cos \frac{2 \pi x}{l}\right)
$$

When $\alpha>1$, the overlying load exceeds the limit load of pillar, the overall instability will occur. When $0<\alpha<1$, the creep failure will occur under the effect of overlying load.

The analysis on the transverse deformation of pillar under different conditions was carried out. The threedimensional distribution map of pillar transverse 
deformation under overlying load, $\alpha$ and pillar height when $f_{0}=500 \mathrm{~mm}$ is shown in Fig.2. It can be seen from the Fig. 2 that transverse deformation distributes symmetrically along the height of pillar, which is the largest in the middle part and the value is $5 \mathrm{~mm}$. With the increasing of $\alpha$, the transverse deformation of the pillar increases nonlinearly.

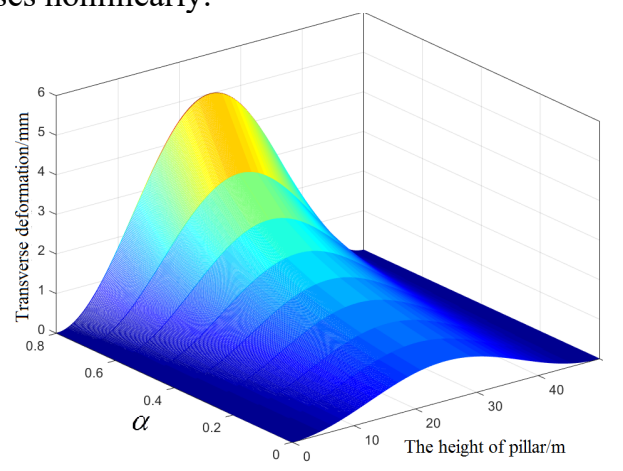

Fig.2. Three-dimensional distribution map of transverse deformation under overlying load, $\alpha$ and pillar height

The three-dimensional distribution map of pillar transverse deformation under overlying load, $f_{0}$ and pillar height when $\alpha=0.3$ is shown in Fig.3. It can be seen from the Fig.3 that transverse deformation distributes symmetrically along the height of pillar, which is the largest in the middle part and the value is $2.8 \mathrm{~mm}$. With the increasing of $f_{0}$, the transverse deformation of the pillar increases linearly.

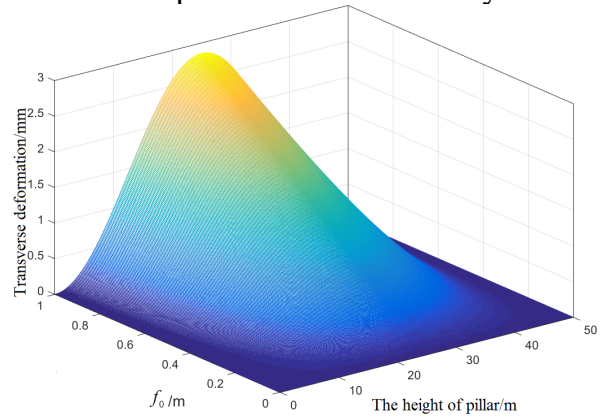

Fig.3. Three-dimensional distribution map of transverse deformation under overlying load, $f_{0}$ and pillar height

The three-dimensional distribution map of the largest transverse deformation under overlying load, $f_{0}$ and $\alpha$ is shown Fig. 4. It can be seen from the Fig.4 that, when $\alpha$ maintained, with the increase of $f_{0}$, the largest transverse deformation increases linerly, the greater the $\alpha$ is, the greater the growth rate is. When $f_{0}$ maintained, with the increase of $\alpha$, the largest transverse deformation increases nonlinerly.

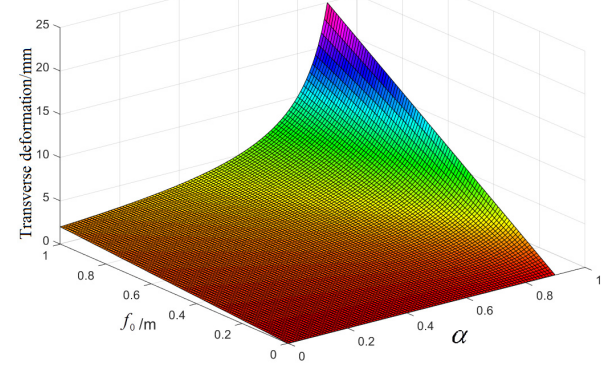

Fig.4. The largest transverse deformation under overlying load,

$$
f_{0} \text { and } \alpha
$$

The influence of $\alpha$ on largest transverse deformation under overlying load of pillar is more intense. With the increase of $f_{0}$, the influence degree becomes larger.

(2) Analysis of stress

Because of the existence of initial transverse deformation, axial and bending deformation will appear in ultrahigh pillar. Therefore, there are two kinds of stress in pillar internal which are separatly axial stress and bending stress.

During the deformation of pillar, the internal stress of pillar can be expressed as

$$
\sigma=\sigma_{1}+\sigma_{0}
$$

Where, $\sigma_{0}$ is the axial stress, $\sigma_{1}$ is the bending stress. Based on the geometric equation, bending strain in pillar can be obtained as

$$
\varepsilon_{1}=\kappa y_{0}=w^{\prime \prime}(x) y_{0}
$$

Where, $\kappa$ is axis curvature, $y_{0}$ is distance from any point to the neutral axis.

Thus, there is a close relationship between the stress and transverse deformation of the pillar. Based on the equation of transverse deformation of ultrahigh pillar, the stress can be obtained. Taking Eq. (9) into Eq. (11), the strain of any point in the pillar can be obtained as

$$
\varepsilon_{1}=\frac{1}{1-\alpha} \frac{4 \pi^{2}}{l^{2}} f_{0} \cdot \cos \frac{2 \pi x}{l} y_{0}
$$

Then the $\sigma_{1}$ can be calculated as

$$
\sigma_{1}=E \varepsilon_{1}=\frac{1}{1-\alpha} \frac{4 \pi^{2} E}{l^{2}} f_{0} \cdot \cos \frac{2 \pi x}{l} y_{0}
$$

The stress of any point in the pillar can be expressed as

$$
\sigma=\frac{1}{1-\alpha} \frac{4 \pi^{2} E}{l^{2}} f_{0} \cdot \cos \frac{2 \pi x}{l} y_{0}+\frac{\alpha P_{c r}}{2 b h_{0}}
$$

\section{Analysis of damage process}

The rock in the concave of the section of the middle of pillar begin to yield, which is shown in Fig.5. Due to the axial compressive stress is larger than bending stress, this paper only considered the plastic yield zone shown in Figure 5. Assuming that axial compressive stress caused by the overlying load maintained in the analysis process. 


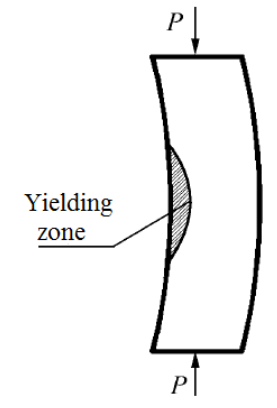

Fig.5. Schematic diagram of plastic area

\subsection{Incubation time of damage of pillar}

When $P<P_{c r}$, the stress in the concave of the section of the middle of pillar can be calculated as

$$
\sigma^{h_{0}}=\frac{1}{1-\alpha} \frac{4 \pi^{2} E}{l^{2}} f_{0} \bullet h_{0}+\frac{\alpha P_{c r}}{2 b h_{0}}
$$

When $\sigma^{h_{0}}>\left[\sigma_{c}\right]$, damage starts to appear.

Based on the Kachanov creep theory[15], the creep damage model for rock can be expressed as:

$$
\dot{D}=C \tilde{\sigma}^{n}=C\left(\frac{\sigma}{1-D}\right)^{n}
$$

Where, $\tilde{\sigma}$ is effective stress; $C$ is material constants; $\dot{D}$ is Change rate of rock damage factor. Taking $t: 0 \sim t, \quad D: 0 \sim D$, based on Eq. (16) using integral, the damage variable evolution equation is obtained as

$$
D=1-\left(1-\frac{t}{t_{I}}\right)^{\frac{1}{n+1}}
$$

Where, $t_{I}$ is time of fracture occurrence, $\mathrm{D}$ is damage factor. Then, $t_{I}$ can be obtained as

$$
t_{I}=\frac{1}{C(n+1)}\left(\frac{1}{\sigma}\right)^{n}
$$

Taking Eq. (15) into Eq. (18), the incubation time can be obtained as

$$
t_{I}=\frac{1}{C(n+1)}\left(\frac{1}{\frac{1}{1-\alpha} \frac{4 \pi^{2} E}{l^{2}} f_{0} \cdot h_{0}+\frac{\alpha P_{c r}}{2 b h_{0}}}\right)^{n}
$$

When $t=t_{I}$, the concave of the section of the middle of pillar begin to damage.

\subsection{Delay time of failure}

After $t>t_{I}$, the damage zone will be gradually extended to the interior. Assuming that the plane sections" hypothesis is still applicable in the process of damage. Setting distance of neutral axial moving to internal is $\Delta \delta$, the thickness of fracture layer is $2 \Delta \delta$, which is shown in Fig.6.

Damage zone front

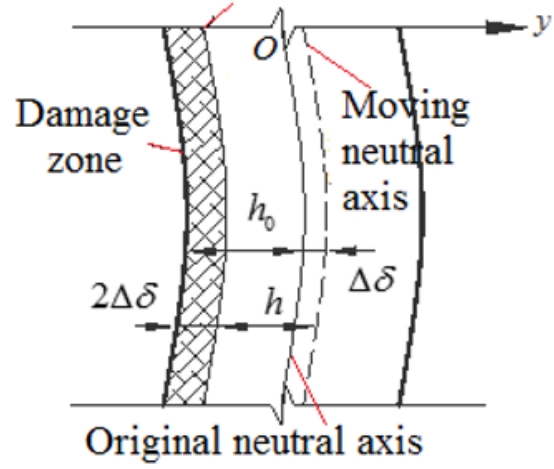

Fig.6. Extension of creep cracks

With reference to Fig. 6 we can see that the distance of fracture layer front to moving neutral axis is $h$, which can be expressed as $h=h_{0}-\Delta \delta$.

Setting $\tau>t_{I}$, then the distance of fracture layer front to moving neutral axis is $h(\tau)$, The $y$-coordinate of any point $P$ in front of fracture layer is $y_{0}$, then the distance between $P$ and moving neutral axis can be obtained as

$$
y(\tau)=y_{0}+\delta=y_{0}+h_{0}-h(\tau)
$$

Assuming $t>\tau$, at the same time the fracture layer front reaches the position of $P$. At this time $y(t)=h(t)$, namely, $h(t)=y_{0}+\delta$, which also can be expressed as

$$
h(t)=h_{0}-\delta=h_{0}-\left[h(t)-y_{0}\right]
$$

Based on the Eq. (20) and Eq. (21), we can obtain as

$$
y(\tau)=2 h(t)-h(\tau)
$$

To solve the derivatives of both sides of Eq. (22), the Eq. (23) can be calculated as

$$
\frac{d^{2} h}{d t^{2}}+\frac{2(n-1)}{h}\left(\frac{d h}{d t}\right)^{2}=0
$$

When $t=t_{I}, h=h_{0}$, at this time $\frac{d h}{d t}=-\frac{h_{0}}{2 t_{I}}$,

Eq.(23) can be solved as

$$
\frac{t}{t_{I}}=1+\frac{2}{2 n-1}\left[1-\left(\frac{h}{h_{0}}\right)^{2 n-1}\right]
$$

Based on the Eq. (15), the maximum compressive stress of time $t$ can be obtained as

$$
\sigma_{c}^{h}=\frac{1}{1-\alpha} \frac{4 \pi^{2} E}{l^{2}} f_{0} \bullet h+\frac{\alpha P_{c r}}{2 b h_{0}}
$$

When $\sigma_{c}^{h}=\left[\sigma_{c}\right]\left(\left[\sigma_{c}\right]\right.$ is ultimate compressive strength), the pillar instability occurs. The elapsed time can be obtained as

$$
t_{\Pi}=\frac{2 n+1-2\left(\sigma^{h_{0}} /\left[\sigma_{c}\right]\right)^{n-1 / 2}}{C(n+1)(2 n-1)\left(\sigma^{h_{0}}\right)^{n}}
$$

Where, $t_{I I}$ is the delay time of failure. 


\section{Engineering case analysis}

\subsection{Engineering background overview}

Dong guashan is a famouse copper mine in China, the mining depth has been greater than $1000 \mathrm{~m}$. In order to improve the efficiency of mining, large-diameter longhole blasting-stage room and subsequent filling mining method is the main mining method, the stope height is always greater than $80 \mathrm{~m}$. Therefore, heightwidth ratio is usually very large, buckling deformation and instability are easy to occur in the pillar under the overlying load. The material parameters and mechanical parameters of the rock in the mining area can be obtained by the actual measurement. The rock material $n=3$ and $C=1.99 \times 10^{-31} \mathrm{~Pa}^{-n} \cdot \mathrm{s}^{-1}$. Ultimate compressive strength $\left[\sigma_{c}\right]$ is $80 \mathrm{MPa}$. Elastic modulus $E$ is $19.5 \mathrm{GPa}$, the pillar width is $4 \mathrm{~m}$, the pillar legth is $8 \mathrm{~m}$ and the pillare height $l$ is $80 \mathrm{~m}$. The depth is $1000 \mathrm{~m}$, The average density $\rho$ of overlying strata is $3.2 \mathrm{~g} \cdot \mathrm{mm}^{-3}$, initial transverse deformation $f_{0}$ is $200 \mathrm{~mm}$.

\subsection{Calculation of damage and instability time}

Limit load of pillar can be obtained as

$P_{c r}=\frac{4 \pi^{2} E I}{l^{2}}=\frac{4 \times 3.14^{2} \times 19500 \times 10^{6} \times 42.67}{80^{2}}=5.13 \times 10^{6} \mathrm{KN}$

The overlying load is calculated as

$P=\rho g h s=3200 \times 10 \times 1000 \times 32=1.02 \times 10^{6} \mathrm{KN}$

Then $\alpha=P / P_{c r}=0.20$.

Based on Eq. (18) the incubation time of damage can be calculated as $t_{I}=18.57 \mathrm{~d}$, delay time of failure can be calculated as $t_{\mathrm{U}}=22.86 \mathrm{~d}$. From the beginning of the damage to overall instability, the filling must be carried out.

The influence of various factors on the $t_{I}$ and $t_{\mathrm{U}}$ is studied in this paper.

\subsection{Sensitivity analysis of influencing factors}

(1) Influencing law of $f_{0}$

The relation curve between $t_{I}, t_{\mathrm{U}}$ and $f_{0}$ is shown in Fig.7. The others parameters are the same as that of engineering case.

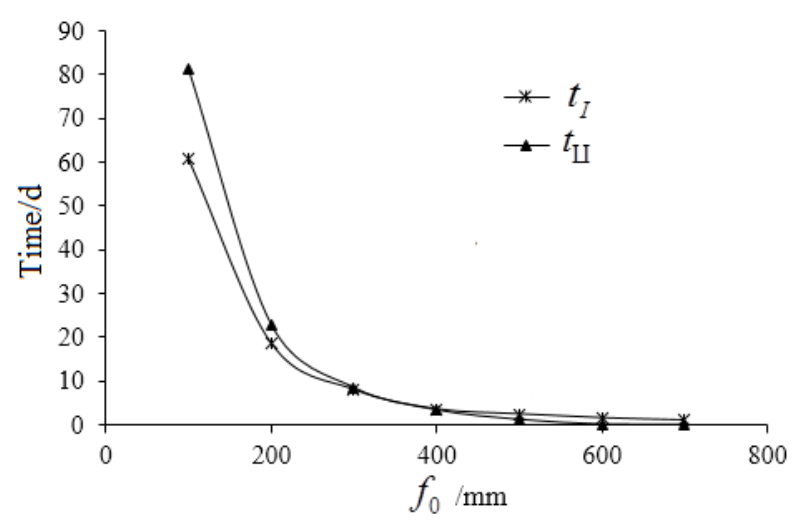

Fig.7. Effect of initial deflection $f_{0}$ on the time of damage

It can be seen from that, with increase of $f_{0}, t_{I}$ and $t_{\amalg}$ will continue to decline. which means that the greater the $f_{0}$ is, the shorter the $t_{I}$ and $t_{\amalg}$ are. After $f_{0}>500 \mathrm{~mm}, t_{\mathrm{U}}$ become 0 . The sudden global instability will occur in the pillar after the appearance of damage. When $f_{0}>800 \mathrm{~mm}$, the pillar cannot keep stable after the formation.

(2) Influence law of $\alpha$

The relation curve between $t_{I}, t_{\mathrm{U}}$ and $\alpha$ is shown in Fig.8. The others parameters are the same as that of engineering case.

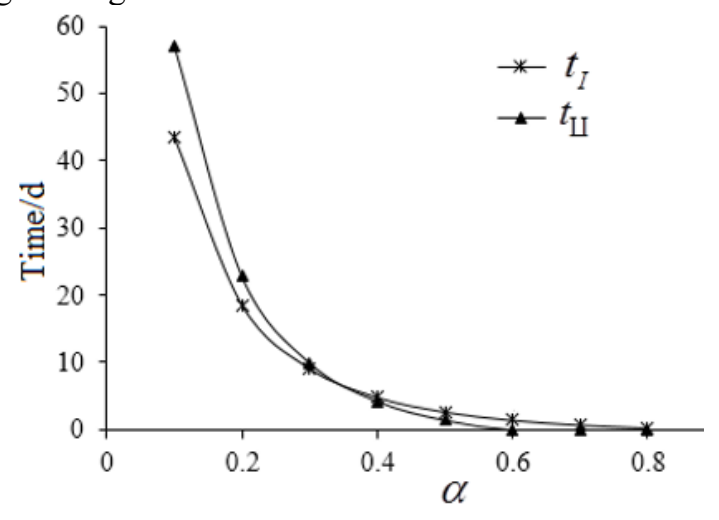

Fig.8. Effect of $\alpha$ on the time of damage

It can be seen from Fig. 8 that the influence law of $\alpha$ is the same as that of $f_{0}$ on the time of damage, delay time of failure. After $\alpha_{>0.6}$, the $t_{I}$ is less than 1 day, the $t_{\amalg}$ become 0 . The sudden global instability will occur in the pillar after the appearance of damage.

(3) Influence law of compressive strength

The relation curve between $t_{I}, t_{\mathrm{U}}$ and $f_{0}$ is shown in

Fig.8. The others parameters are the same as that of engineering case.

It can be seen from Fig.9 that ultimate compressive strength does not affect $t_{I}$ but only have an impact on the $t_{\mathrm{U}}$. The greater the ultimate compressive strength is, 
the greater the $t_{\amalg}$ is, the more stable is, but the less the growth rate is.

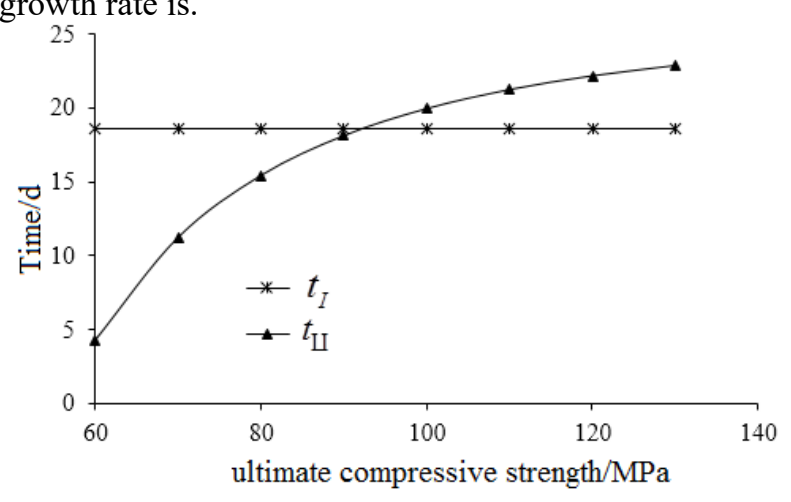

Fig.9 Effect of compressive strength on the damage

\section{Conclusion}

Based on the elastoplastic theory and strut method, this paper established the calculation model of ultrahigh pillar with initial transverse deformation. The transverse deformation of the pillar is analyzed. Then, the damage process and instability time of ultrahigh pillar are analyzed based on the creep theory. The main conclusions are as follows.

(1) For the ideal pillar, when the overlying load is less than the critical load of the pillar, there is only compression deformation but no transverse deformation in the pillar. As for the ultrahigh pillar with initial transverse deformation, the additional transverse deformation will be produced under the action of the overlying load. The research shows that the additional transverse deformation of pillar is closely related with the ratio of overlying load and limit load $(\alpha)$ and initial transverse deformation $\left(f_{0}\right)$. Additional transverse deformation increases linearly with the increasing of initial transverse deformation. With the increasing of ratio of overlying load and limit load, additional transverse deformation increases nonlinearly.

(2) Analysis of engineering examples shows that incubation time of damage $\left(t_{I}\right)$ and delay time of failure ( $t_{\amalg}$ ) are closely related with $f_{0}, \alpha$, ultimate compressive strength. With the increasing of $\alpha$ and $f_{0}$, the $t_{I}$ and $t_{\amalg}$ decrease rapidly. When $\alpha>0.6$ or $f_{0}>500 \mathrm{~mm}, t_{\amalg}$ become 0 and $t_{I}<1$ day, the sudden global instability will occur in the pillar after the appearance of damage. At this time, the control measures should be taken in time, such as filling or strengthening support. The compressive strength mainly affects the $t_{\amalg}$. The greater the ultimate compressive strength is, the greater the $t_{\amalg}$ is.

(3) The initial transverse deformation of pillar, which is caused by the blasting in the mining process, has an important influence on the stability of pillar. Therefore, in order to ensure the stability of the pillar, the disturbance to the pillar should be reduced so as to reduce the $f_{0}$. In addition, with the increase of the mining depth, the overlying load increases and $\alpha$ becomes larger, which results in shorter stability time of pillar. At this time, we should increase the width of the pillar and reduce the height of the pillar to strengthen the stability of pillar.

(4) The results of this study has an important guiding role in the improvement of stability control and management.

\section{Acknowledgement}

Financial supports for this work, provided by the National Natural Science Foundation of China (NO. 51804016) and the General Financial Grant from the China Postdoctoral Science Foundation (No. 2016M591080) are gratefully acknowledged. Finally, the first author would like to thank colleagues at USTB for their support.

\section{References}

1. M. Cravero, G. labichino, O. Del Greco, Experiences in the measurement of stresses and displacements in the Masua mine. Proceedings of the 3rd International Symposium on Field Measurements in Geomechanics. (1991)

2. Anon, Coal mining technology: some british developments. 23, 3, 38-40 (1986)

3. Stepanov Y, Solov V O, Effect of the main parameters of an explosion-reactive unit (ERU) on the size of the cavity formed in soft rock. Soviet Mining Science 26, 2, 154-158 (1991)

4. Sakamoto Akio, Yamada Noritoshi, Iwaki Keisuke, Applicability of recycling materials to cavity filling materials. Journal of the Society of Materials Science 54, 11, 1123-1128 (2005)

5. Whittles. D.N, Lowndes. I. S, The stability of methane capture boreholes around a long wall coal panel. International Journal of Coal Geology 71, 2, 313-328 (2007)

6. Bahuquna. P.P, Subsidence studies in Indian coalfields by a semi-empirical approach. IAHSAISH Publication 71,234, 127-133 (1995)

7. Peng Cideng, Topical areas of research need in ground control: a state of the art review on coal mine ground control. Journal of China University of Mining\& Technology 44, 1, 1-8 (2015)

8. Wu Hai, Zhang Nong, Wang Weijun, Characteristics of deformation and stress distribution of small coal pillars under leading abutment pressure. International Journal of Mining Science and Technology 25, 6, 921-926 (2015)

9. Wang Jinan, Li Dazhong, Shang Xinchun, Mechanics analysis on creep fracture of strong roof strata above mined-out area. Journal of University of Science and Technology Beijing 33, 2, 142-148 (2011) 
10. He Guangling, Li Duchun, Zhai Zhiwen, Analysis of instability of coal pillar and stiff roof system. Journal of China coal society 32, 9, 897-901 (2007)

11. Li Hong, $\mathrm{Xu} Z$ Zenghe, $\mathrm{Xu}$ Xiaohe, Criterions and forewarning of ore pillar rock bursts in asymmetric mining. China Mining Magazine 6, 1, 46-51 (1997)

12. Exadaktylos G, Tsoutrelis C E, Pillar failure by axial splitting in brittle rocks. Int. J. Rock Mech. Min. Sci. and Geomech. Abstr. 32, 6, 551-562 (1995)

13. Yin Shenghua, WU Aixiang, LI Xiwen, Orthogonal polar difference analysis for sensitivity of the factors influencing the ore pillar stability. Journal of China Coal Society 37, 47-52 (2012)

14. FU Jianxin, Evolution mechanism of damage of goaf in deep hard rock mines and its stability control. University of Science and Technology Beijing (2015)

15. Xianhe Du, Donghuan Liu, Yinghua Liu, Numerical Limit Load Analysis of 3D Pressure Vessel with Volume Defect Considering Creep Damage Behavior. Mathematical Problems in Engineering 2015, 1-12 (2015) 